\title{
Systemic Perfusion: a Method of Enhancing Relative Tumor Uptake of Radiolabeled Monoclonal Antibodies
}

\author{
RICHARD L. WAHL,* CYNTHIA R. PIKO, BARBARA A. BEERS, \\ ONELIO GEATTI, JON JOHNSON and PHIL SHERMAN
}

\begin{abstract}
Division of Nuclear Medicine, Department of Internal Medicine, University of Michigan Medical Center.
\end{abstract} Ann Arbor, MI 48109-0028, U.S.A.

(Received 14 April 1988)

\begin{abstract}
We evaluated the feasibility of systemic vascular perfusion with saline (mimicking plasmapheresis) as a method to enhance tumor-specific monoclonal antibody (MoAb) tumor/background ratios. Initially, groups of rats were injected intravenously (i.v.) with ${ }^{13 i} \mathrm{I}-5 \mathrm{G} 6.4 \mathrm{MoAb}$ (murine IgG2aK reactive with ovarian carcinoma). These animal's radioactivity levels were determined by dose calibrator and they were imaged before and after perfusion which was conducted at 4 or $24 \mathrm{~h}$ post-antibody injection. Animals were sacrificed after perfusion, as were controls, and normal organ radioactivity levels determined. In addition, nude mice bearing HTB77 ovarian cancers subcutaneously were injected i.v. with ${ }^{131} \mathrm{I}-5 \mathrm{G} 6.4 \mathrm{MoAb}$ and were imaged before and after systemic perfusion with saline 24 h post-5G6.4 injection. Perfusion in rats dropped whole-body 5 G6.4 levels significantly at both perfusion times $(P<0.0005)$. The drop in whole-body radioactivity with perfusion was significantly greater for the animals perfused at $4 \mathrm{~h}$ post i.v. 5 G6.4 antibody injection $(48.3 \pm 5.1 \%)$ than for those perfused at $24 \mathrm{~h}$ post i.v. antibody injection $(32.9 \pm 2.9 \%)(P<0.025)$. In the nude mice with ovarian cancer xenografts, $\gamma$ camera images of tumors were visually and quantitatively (by computer image analysis) enhanced by perfusion, with a 2.33 -fold greater decline in whole body uptake than in the tumor $(P<0.05)$. These studies show that $(1)$ much background antibody radioactivity can be removed using whole-body perfusion with saline, (2) that the decline in whole body activity is larger with 4 than $24 \mathrm{~h}$ perfusion and (3) tumor imaging can be enhanced by this approach. This and similar approaches that increase relative tumor antibody uptake such as plasmapheresis may be useful in imaging and therapy with radiolabeled antibodies.
\end{abstract}

\section{Introduction}

When intact monoclonal antibodies (MoAbs) are injected intravenously, peak absolute tumor uptakes of antibody are in the first 12 days post-injection (Goodwin, 1987). Imaging is not optimal until considerably later, as elevated levels of blood pool radioantibody persist for days following i.v. intact antibody injection, and the resulting relatively low tumor/background ratios detract from the utility of intact MoAbs as imaging and therapeutic agents (Goodwin, 1987). Only as blood pool radioantibody levels clear do tumor/background ratios improve. Computer background subtraction methods, while improving diagnostic sensitivity in imaging studies, do not impact upon these low target/background ratios for purposes of radioimmunotherapy (Goldenberg et al., 1978; Wahl et al., 1986b). While

* Reprint requests and correspondence should be addressed to: Richard L. Wahl, M.D., University of Michigan Medical Center, Division of Nuclear Medicine, $1500 \mathrm{E}$. Medical Center Drive, Ann Arbor, MI 48109-0028, U.S.A. [Tel: (313) 936-5384]. antibody fragments do significantly enhance tumor/background ratios and allow for imaging at earlier time points than intact antibody, they result in less absolute radioantibody reacting the tumor than if intact antibody is used (Wahl et al., 1983). Alternative approaches to improving target/background ratios, using polyclonal anti-mouse antibodies (either attached to liposomes or alone) to clear the primary radiolabeled antibody from the blood after maximal tumor uptake has been achieved, have been investigated, but can result in high levels of splenic and to a lesser extent hepatic activity (Begent et al., 1982; Sharkey et al., 1985).

If it were possible to transiently have high blood levels of tumor-specific antibody following i.v. injection, allowing antibody to accumulate in tumor, and then to rapidly remove blood pool radioantibody without increasing reticuloendothelial system uptake, it might be possible to considerably enhance relative tumor uptake. This would obviously enhance imaging and increase the possibility of successful radioimmunotherapy with radiolabeled antibody. In the present study we evaluated the feasibility 
of enhancing specific antibody tumor/background ratios by experimentally attempting to achieve the total removal of circulating blood pool ratioantibody from normal and tumor-bearing animals by exhaustive perfusion with saline. While this approach is not compatible with life, such an approach, if enhancing tumor/background ratios, would lay a logical framework for the more selective removal of radiolabeled MoAbs from the blood, such as immuno-adsorption plasmapheresis, which can be performed in the living (Terman et al., 1979).

\section{Methods}

\section{Monoclonal antibody}

5G6.4 is an IgG2ak murine MoAb reactive with ovarian and other epithelial carcinomas (Wahl et al., 1986a). It localizes specifically to ovarian cancer xenografts when administered intravenously (Wahl et al., 1985a). It was grown in Balb/c micc as ascites and purified by staphylococcal protein A chromatography (Ey et al., 1978). Purity was assured by SDS PAGE gels in the reduced and unreduced state (Laemmli, 1970). The antibody was labeled by the iodogen (Pierce Chemical-Rockford, Ill.) method using a 5-8/1 antibody/iodogen ratio. Specific activity of the injected preparations was approximately $6 \mu \mathrm{Ci} / \mu \mathrm{g}$. Separation of free from bound iodine was by Biogel P-60 sizing chromatography. Greater than $95 \%$ freedom from contaminating iodine was demonstrated by silica gel TLC (Wahl et al., 1985b). Binding of the labeled protein to target cells was demonstrated by a $1 \mathrm{~h}$ direct binding assay to HTB77 target cells (Wahl et al., 1985b; Lindmo et al., 1984).

\section{Animal studies}

Healthy female Sprague-Dawley rats were used in the rat studies, while athymic Swiss nude mice with HTB77 s.c. ovarian carcinoma xenografts (which occur 3-6 weeks post the s.c. injection of 10 million HTB77 cells) were used in the tumor-bearing animal studies. Four groups of five rats were injected i.v. in the femoral vein following cut down, with approximately $19 \mu \mathrm{Ci}$ of ${ }^{131} \mathrm{I}-5 \mathrm{G} 6.4$. Perfusion of the normal rats (which were under ether anesthesia during the study) was conducted by cannulation of the femoral vein with a butterfly needle, and slowly flushing with heparinized saline (approximately $100 \mathrm{~cm}^{3}$ of perfusate was used), with drainage of blood from the opposite femoral vein. Thus all organs, except the injected leg, were well-perfused. The animals werc perfused until the effluent was nearly clear. Groups of five rats were perfused 4 or $24 \mathrm{~h}$ following i.v. antibody injection, with images obtained before and after perfusion. Animals' whole-body radioactivity level was also assessed in a dose calibrator both before and after systemic perfusion. Control groups of unperfused animals were also sacrificed at 4 and $24 \mathrm{~h}$ following antibody injection. Radioactivity levels/g of injected antibody in selected tissues were determined using standard tissue counting methods (Wahl et al., 1984). In nude mice with HTB77 tumors cstablished subcutaneously, the ${ }^{131} \mathrm{I}-5 \mathrm{G} 6.4$ was injected i.v., and $24 \mathrm{~h}$ later perfusion was performed by removal of circulating blood, by cannulation and saline perfusion of the right ventricle with drainage of blood from the tail vein, as well as subsequent left ventricular injection with removal of blood from the right heart. Gamma camera imaging was performed using a GE400 AT $y$ camera attached to a STAR computer before and after perfusion, using a $128 \times 128$ image matrix, word mode, a fixed imaging time of $10 \mathrm{~min}$ and a fixed $\gamma$ camera-to-animal imaging distance. Regions of interest for whole body with and without tumor were selected and analyzed. A high energy parallel hole collimator was used. Statistical analysis was by ANOVA, the paired- $t$ (when the animal was used as its own control) and the unpaired $t$-tests.

\section{Results}

Systemic perfusion in rats was somewhat difficult initially and even with practice could require $20 \mathrm{~min}$ or longer per rat to complete. Difficulties with leakage at the injection site were not uncommon, but could largely be avoided by keeping the infusion rate slow. The perfusion studies in the nude mice were far more difficult than in the rat, as vascular access was more difficult, and it was not possible to get as complete a degree of perfusion in these animals. In spite of some of the technical difficulties, it was possible in the rats to get quite complete perfusion, as judged by the nearly "clear" fluid removed from the opposite femoral vein by the conclusion of the perfusion, indicating that nearly all of the easily accessible blood pool had been exchanged for saline. Such complete perfusion was not achieved in the mice, however.

Whole-body activity in the rats as assessed by dose calibrator reading of the entire animal before and after perfusion showed a clear drop when perfusion was done at $4 \mathrm{~h} \quad(17.7 \pm 0.14-9.14 \pm 0.94 \mu \mathrm{Ci}$, mean \pm SEM $)(P<0.005)$ or at $24 \mathrm{~h}(13.18 \pm 0.21$ $8.86 \pm 0.47 \mu \mathrm{Ci}) \quad(P<0.05)$. The post-perfusion whole-body activities as a percent of pre-perfusion values $(4 \mathrm{~h}$ post $/ \mathrm{pre}=51.7 \pm 5.1 \%$ vs $67.1 \pm 2.9 \%)$ indicated that the animals perfused $4 \mathrm{~h}$ post-injection had a significantly greater decline in whole-body activity than animals perfused at $24 \mathrm{~h}$ post i.v. injection $(P<0.25)$. Representative $\gamma$ camera images of several of the rats before [Fig. 1(a)] and after [Fig. 1(b)] perfusion additionally show this decline in whole-body activity, in this case with perfusion performed at $4 \mathrm{~h}$ post i.v. antibody injection.

The percent of injected radioactivity detected/g of tissue at sacrifice in the control and perfused groups are shown in Table 1. Note that when perfusion was conducted at $4 \mathrm{~h}$ post i.v. antibody injection, the 

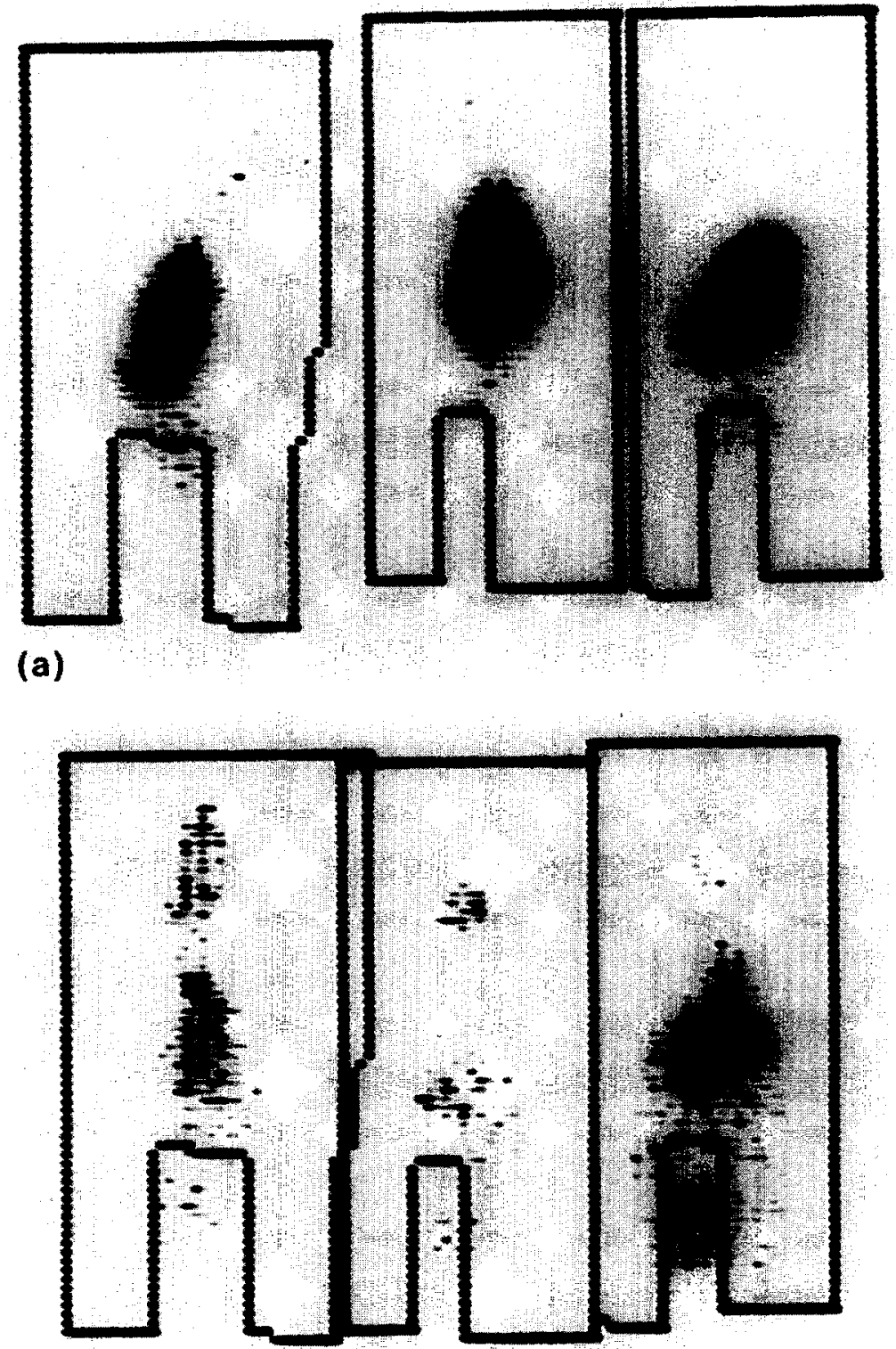

(b)

Fig. 1. (a) Gamma camera image $3 \frac{1}{2} \mathrm{~h}$ post i.v. injection of $19 \mu \mathrm{Ci}$ of ${ }^{131} \mathrm{I}-5 \mathrm{G} 6.4$ antibody. Three rats are shown. (b) Immediately after whole body perfusion at $4 \mathrm{~h}$ post-i.v. injcetion. Note the considerably decreased whole body activity in (b). In five animals the mean post-perfusion whole-body activity was $51.7 \pm 5.1 \%$ of the pre-perfusion activity. 
(a)

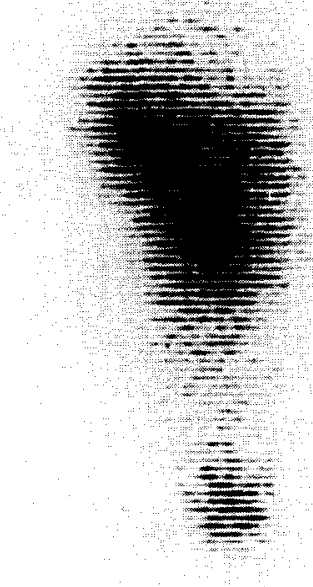

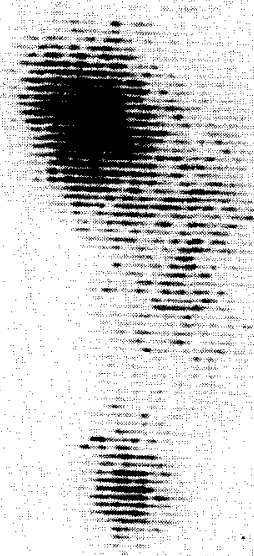

(b)

Fig. 2. (a) Twenty four hours post i.v. injection image of a representative nude mouse with a s.c. ovarian carcinoma xenograft in the left shoulder region. Considerable whole-body background activity is present. (b) Immediately after perfusion, far clearer visualization of the tumor relative to background is seen. Activity in the lower portion of the animal is in the urinary bladder. In the group of animals studied, the drop in whole-body radioactivity was $28.5 \pm 7.1 \%$ while the drop in tumor radioactivity was 2.33 -fold less $(12.1 \pm 5.7 \%)(P<0.05)$ based on computer quantitation of $\gamma$ camera images 
Table 1. Normal rat perfusion vs controls (a) Sacrifice $4 \mathrm{~h}$ post antibody injection

\begin{tabular}{lcc}
\hline & \multicolumn{1}{c}{ Controls } & 4h Perfusion \\
\hline Liver & $0.133 \pm 0.007$ & $0.054 \pm 0.007^{*}$ \\
Kidney & $0.187 \pm 0.011$ & $0.050 \pm 0.006^{*}$ \\
Spleen & $0.090 \pm 0.002$ & $0.036 \pm 0.004^{*}$ \\
Lung & $0.316 \pm 0.044$ & $0.093 \pm 0.011^{\star}$ \\
Leg muscle & $0.007 \pm 0.000$ & $0.019 \pm 0.007 \dagger$ \\
S. bowel & $0.126 \pm 0.006$ & $0.042 \pm 0.006^{*}$ \\
Blood & $0.763 \pm 0.083$ & $\mathrm{ND}$ \\
\hline
\end{tabular}

* Significantly $(P<0.0005)$ less than control values. + Not perfused due to design of system.

(b) Sacrifice $24 \mathrm{~h}$ after i.v. antibody injection

\begin{tabular}{lll}
\hline & \multicolumn{1}{c}{ Controls } & 24 h Perfusion \\
\hline Liver & $0.065 \pm 0.002$ & $0.038 \pm 0.004^{* * * *}$ \\
Kidney & $0.078 \pm 0.003$ & $0.050 \pm 0.013$ \\
Spleen & $0.042 \pm 0.001$ & $0.026 \pm 0.004^{* *}$ \\
Lung & $0.197 \pm 0.027$ & $0.106 \pm 0.020$ \\
Leg muscle & $0.014 \pm 0.001$ & $0.011 \pm 0.001 \dagger$ \\
S. bowel & $0.057 \pm 0.003$ & $0.034 \pm 0.004^{* * *}$ \\
Blood & $0.420 \pm 0.027$ & $\mathrm{ND}$ \\
\hline
\end{tabular}

****P<0.005 less than control values; ***P<0.01 less than control values; $* * P<0.025$ less than control values

Mean \pm SEM post-perfusion and controls; $(n=5$ animals/group); results: $(\% \mathrm{~kg}$ dose/g).

declines in organ activity/g are significant at the $P<0.005$ level (leg muscle was not perfused). Drops in tissue activity at $24 \mathrm{~h}$ following perfusion, relative to controls, were slightly less marked, though still significant ( $P$ in $0.01-0.1$ range). The liver activity drop with $4 \mathrm{~h}$ perfusion $(72 \%)$ is greater than with $24 \mathrm{~h}$ pcrfusion $(41 \%)(P<0.01)$, compatible with the dose calibrator data.

Having demonstrated a $33-50 \%$ drop in wholebody activity and a $50-70 \%$ drop in normal organ activities in rats following systemic perfusion, the technique was then extended to nude mice with s.c. ovarian tumors present. This was considerably more demanding technically, as it was far more difficult to achieve satisfactory vascular access in the nude mice and thus the perfusion was less complete. After developing a somewhat optimized technique, the feasibility of the technique was tested in a group of nude mice which had HTB77 s.c. ovarian tumors, These animals were injected intravenously with $30 \mu \mathrm{Ci}$ of ${ }^{131}$ I-5G6.4 antibody. Twenty-four hours later they were imaged for identical time periods before and after perfusion with saline using a $\gamma$ camera with data recorded into a computer. Representative images using the same imaging parameters, are shown before [Fig. 2(a)] and after [Fig. 2(b)] perfusion. Tumor visualization is considerably enhanced in the post-perfusion images, despite the fact that there is only a $28.5 \pm 7.1 \%$ drop in whole-body activity (based on whole body ROI, less tumor, mean \pm SEM of 4 animals pre- and post-perfusion) indicating that perfusion was probably incomplete relative to the rat data. The image enhancement apparent in the animal was due to the fact that tumor uptake dropped only $12.1 \pm 5.7 \%$ (based on tumor ROI data), considerably less than the whole-body activity drop $(P<0.05)$ resulting in improved tumor/non-tumor ratios.

\section{Discussion}

These feasibility studies clearly demonstrate that exhaustive perfusion with saline in the rat and less exhaustive perfusion in the nude mouse with ovarian carcinoma xenografts removes a significant amount of whole body radioantibody activity and enhances tumor imaging. Using this approach in rats, drops of up to $72 \%$ in liver activity were achieved with perfusion at $4 \mathrm{~h}$ and $41 \%$ at $24 \mathrm{~h}$ following i.v. antibody injection. While the drops in whole animal activity were less in the normal mice (due to the difficulty of the procedure) than in the rats, they were sufficient to considerably enhance tumor imaging by improving quantitative tumor/background ratios on $\gamma$ scans. This enhancement occurred as tumor activity did not decline as much as whole-body activity with the antibody removal.

The normal tissue activities dropped more at $4 \mathrm{~h}$ vs controls than they did after $24 \mathrm{~h}$ of the antibody circulating systemically. This was also true in the dose calibrator studies comparing the drop in whole-body activity in the experimental animals pre- and postperfusion. This lesser drop, with equal amount of perfusate, is most likely due to the slow diffusion of radioantibody out of the immediately exchangeable blood pool following i.v. administration. Presumably less circulating antibody is easily accessible at later times. Consequently there was more difficulty removing antibody from the entire animal at 24 than $4 \mathrm{~h}$. It is probable that even larger drops in systemic radioantibody levels could be achieved if the perfusion could be carried out over a longer period of time, to enhance the transit of radioantibody from the extravascular to the vascular compartment, with subsequent removal of activity from the blood vessels. The magnitude of such enhancement, and the effect on tumor uptake, would need further evaluation as it is probable that tumor radioactivity levels might drop, in time, as well.

It appears that the mechanism of the enhancement in tumor/non-tumor ratios by perfusion is due to the fact that specific antibody is retained at the tumor due to binding to the antigen in the tumor while blood pool antibody levels are more greatly reduced through perfusion. It is, of course, possible that some of the effect may be due to slower transit of antibody out of tumor interstices than from normal tissues. Additional studies, including dual-label studies would be necessary to more definitively address this mechanistic issue, though the fact remains that the lumor/background ratios are, at least acutely, enhanced by perfusion. Studies in which the animals can live for a longer period of time following removal of circulating blood pool activity will also be necessary to evaluate the effect on whether the cumulative dose of radioactivity to tumors following the systemic 
clearance is enhanced relative to the whole-body dose.

While some loss of tumor radioactivity was seen in the quantitative $\gamma$ images with the perfusion procedure, it was less than whole body losses, indicating that tumor retention of antibody was greater than whole-body retention. This is the key to the technique: the specific antibody remains associated with the tumor, but is washed away to a greater extent from normal tissues. We have recently applied a conceptually similar technique to lavage of specific antibody away from normal structures in the peritoneal cavity, but have seen retention at the tumor (Wahl et al., 1987b).

It is obvious that systemic perfusion in its present form, removal of the circulating blood pool activity by repeated injection with saline with resultant exsanguination, is a technique that is not well-suited to clinical practice. Rather, it is a laboratory demonstration of what might be possible if circulating radioantibody is removed extracorporeally. The technique does not appear to result in high reticuloendothelial system levels of radioactivity, as can be seen with the polyclonal anti-mouse clearance technique, and seems to have considerable promise (Wahl and Fisher, 1987a). Plasmapheresis, and other more selective blood cleansing processes, should have results similar to systemic perfusion and this study strongly suggests that such manipulations should considerably improve target/background ratios, and possibly increase therapeutic indices with radiolabeled antibodies (Terman et al., 1987).

In conclusion, systemic perfusion with saline results in a larger drop of whole-body radioantibody activity than tumor activity. This effect enhances tumor visualization on $\gamma$ scans and should lead to better therapeutic indices for radiolabeled antibodies, if functionally-similar techniques that are bloodsparing, can be applied.

Acknowledgement-Supported by CA 41531 awarded by the NCI-PHS.

\section{References}

Begent, R. H.; Keep, P. A.; Green, A. F., et al. Liposomally entrapped second antibody improves tumor imaging with radiolabelled (first) anti-tumor antibody. Lancet 1(8332): 1047-1048; 1982.
Ey, P.; Prowse, S.; Jenkins, C. Isolation of pure IgG1, IgG2a and $\mathrm{IgG} 2 \mathrm{~b}$ from mouse serum using protein A-Sepharose. Immunochem. 15:429-436; 1978.

Goldenberg, D. M.; DeLand, F.; Kim, E.; Bennett, S.; Primus, F. J.; van-Nagel, J. R.; Estes, N.; DeSimone, P.; Rayburn, P. Use of radiolabeled antibodies to carcinoembryonic antigen for the detection and localization of diverse cancer by external photoscanning. $N$. Engl. $J$. Med. 298(25): 1384-1386; 1978.

Goodwin, D. A. Pharmacokinetics and antibodies. J. Nucl. Med. 28:1358-1362; 1987.

Laemmli, V. K. Clevage of structural proteins during assembly of the head of bacteriophage T4. Nature 222:680-685; 1970.

Lindmo, T.; Boven, E.; Cuttitta, F.; Fedorko, J.; Bunn, P. A. Determination of the immunoreactive fraction of radiolabeled monoclonal antibodies by linear extrapolation to binding at infinite antigen excess. $J$. Immunol. Med. 7(1):77-89; 1984.

Sharkey, R. M.; Primus, F. J.; Goldenberg, D. M. Second antibody clearance of radiolabeled antibody in cancer radioimmunodetection. $P N A S-U S A \quad$ 81:2843-2846; 1985.

Terman, D. S.; Buffaloe, G.; Mattioli, C.; Cook, G.; Tillquist, R.; Sullivan, M.; Ayus, J. C. Extracorporeal immunoadsorption: Initial experience in human systemic lupus erythematosus. The Lancet 1:824-827; 1979.

Wahl, R. L.; Parker, C. W.; Philpott, G. W. Improved radioimaging and tumor localization with monoclonal F(ab')2. J. Nucl. Med. 24:316-325; 1983.

Wahl, R. L.; Sherman, P.; Fisher, S. The effect of specimen processing on radiolabeled monoclonal antibody biodistribution. Eur. J. Nucl. Med. 9:382-384; 1984.

Wahl, R. L.; Geatti, O.; Liebert, M.; Laino, L. Radioimmunoimaging and localization of human ovarian carcinoma xenografts. Radiology 157(P):99; 1985a.

Wahl, R. L.; Liebert, M.; Carey, J. E.; Jackson, G. Quality control of radiolabeled monoclonal antibodies: Immunologic and radiochemical. Cancer Drug Delivery 2(3): 236; 1985 b.

Wahl, R. L.; Liebert, M.; Biesman, B.; Roberts, J.; Jackson, G.; Kronberg, S.; Laino, L. Production and characterization of a murine monoclonal antibody reactive with ovarian and other epithelial carcinomas. PAACR 27:355; 1986a.

Wahl, R. L.; Tuscan, M. J.; Botti, J. M. Dynamic variable background subtraction: A simple means of displaying radiolabeled monoclonal antibody scintigraphy. J. Nucl. Med. 27:454-548; 1986b.

Wahl, R. L.; Fisher, S. Intraperitoneal delivery of monoclonal antibodies: Enhanced regional delivery advantage using intravenous unlabeled anti-mouse antibody. $\mathrm{Nucl}$. Med. Biol. 14(6):611-615; 1987a.

Wahl, R. L.; Fisher, S.; Sherman, P. Intraperitoneal delivery of radiolabeled monoclonal antibodies: Effect of peritoneal lavage on intraperitoneal tumor uptake. J. Nucl. Med. 28(4):715; $1987 \mathrm{~b}$. 